\title{
Hypercatadioptric Line Images for 3D Orientation and Image Rectification
}

\author{
J. Bermudez-Cameo, L. Puig, J.J. Guerrero \\ Robotics, Perception and Real Time Group ( RoPeRT) \\ Instituto de Investigación en Ingeniería de Aragón (I3A) \\ Universidad de Zaragoza, Mariano Esquillor s/n, 50018, Zaragoza, Spain. \\ Tel.+34-976762707,Fax+34-976762043,e-mail: bermudez@unizar.es
}

\begin{abstract}
In central catadioptric systems 3D lines are projected into conics. In this work we present a new approach to extract conics in the raw catadioptric image, which correspond to projected straight lines in the scene. Using the internal calibration and two image points we are able to compute analytically these conics which we name hypercatadioptric line images. We also perform an exhaustive analysis on the elements that can affect the conic extraction accuracy and its error propagation. Besides that, we exploit the presence of parallel lines in man-made environments to compute the dominant vanishing points (VPs) in the omnidirectional image. In order to obtain the intersection of two of these conics we analyze the self-polar triangle common to this pair. With the information contained in the vanishing points we are able to obtain the 3D orientation of the catadioptric system. This method can be used either in a vertical stabilization system required by autonomous navigation or to rectify images required in applications where the vertical orientation of the catadioptric system is assumed. We use synthetic and real images to test the proposed method. We evaluate the 3D orientation accuracy with a ground truth given by a goniometer and with an inertial measurement unit (IMU). We also test our approach performing vertical and full rectifications in sequences of real images.
\end{abstract}

